\title{
Antigen 5 Allergens of Hymenoptera Venoms and Their Role in Diagnosis and Therapy of Venom Allergy
}

\author{
Simon Blank ${ }^{1}$ (D) Murilo Luiz Bazon ${ }^{1,2} \cdot$ Johannes Grosch ${ }^{1} \cdot$ Carsten B. Schmidt-Weber ${ }^{1}$. \\ Márcia Regina Brochetto-Braga ${ }^{2}$. Maria Beatrice Bilò ${ }^{3}$. Thilo Jakob ${ }^{4}$
}

Published online: 9 July 2020

(C) The Author(s) 2020

\begin{abstract}
Purpose of Review Stings of Hymenoptera of the superfamily Vespoidea such as yellow jackets, paper wasps or stinging ants are common triggers for severe and even fatal allergic reactions. Antigen 5 allergens are potent allergens in the majority of these venoms with major importance for diagnosis and therapy. Reviewed here are the characteristics of antigen 5 allergens, their role in component-resolved diagnostics as well as current limitations of the available diagnostics for proper therapeutic decisions. Recent Findings Antigens 5 are proteins of unknown function in Hymenoptera venoms with high allergenic potency. They represent key elements in component-resolved diagnosis to discriminate between honeybee and vespid venom allergy. However, due to their pronounced cross-reactivity, there are remaining diagnostic and therapeutic challenges that have to be addressed. Summary Antigens 5 are highly relevant venom allergens of the Vespoidea superfamily. Although their use in componentresolved diagnosis facilitates dissection of cross-reactivity and primary allergy in double sensitization to honeybee and vespid venom, new diagnostic concepts are needed to discriminate between allergies to different vespid species.
\end{abstract}

Keywords Antigen $5 \cdot$ Component-resolved diagnostics $\cdot$ Allergen cross-reactivity $\cdot$ Hymenoptera venom allergy $\cdot$ Polistes dominula venom $\cdot$ Yellow jacket venom

\section{Introduction}

Stings of Hymenoptera are one of the most frequent triggers for severe IgE-mediated anaphylaxis in adults [1]. Systemic

Simon Blank and Thilo Jakob contributed equally to this work.

This article is part of the Topical Collection on Allergens.

Simon Blank

simon.blank@tum.de

1 Center of Allergy and Environment (ZAUM), School of Medicine and Helmholtz Center Munich, German Research Center for Environmental Health, Member of the German Center of Lung Research (DZL), Technical University of Munich, Ingolstädter Landstraße 1, 85764 Munich, Germany

2 Department of General and Applied Biology, Biosciences Institute, Sao Paulo State University, Rio Claro, São Paulo, Brazil

3 Department of Clinical and Molecular Sciences, Ancona and Allergy Unit, Department of Internal Medicine, University Hospital of Ancona, Polytechnic University of Marche, Ancona, Italy

4 Experimental Dermatology and Allergy Research Group, Department of Dermatology and Allergology, Justus-Liebig-University Gießen, Giessen, Germany reactions to the venoms of stinging Hymenoptera may be restricted to generalized symptoms of the skin, but can also affect the respiratory and vascular system and lead to multiorgan failure. Fatal anaphylaxis after Hymenoptera stings is a rare but well-recognized cause of sudden death [2] and accounts for approximately $20 \%$ of cases of anaphylaxis-related fatalities [3]. Hymenoptera venom allergy can be effectively treated by venom-specific allergen immunotherapy (VIT), which represents the only available curative treatment. Efficacy and safety of VIT highly depend on the unequivocal identification of the culprit insect causing clinical symptoms and, hence, the correct choice of venom for therapy. VIT was reported to be effective in preventing subsequent systemic sting reactions in $77-84 \%, 91-96 \%$ and $97-98 \%$ of patients allergic to honeybee venom (HBV), yellow jacket venom (YJV) and ant venom, respectively [4].

Allergy-relevant Hymenoptera belong to the superfamilies of Apoidea and Vespoidea. Honeybees (Apis mellifera) are elicitors of venom allergy in areas all over the world and also yellow jackets (Vespula spp.) are common allergy-relevant species, particularly in the Northern hemisphere, whereas paper wasps are of greater importance in the US (e.g. $P$. annularis, $P$. exlamans) and the Mediterranean region of 
Europe (Polistes dominula). In South America, other Polistinae such as Polybia, Agelaia and Apoica are of special importance. Moreover, venom allergy can be caused by stings of bumblebees (Bombus spp.) and hornets (Vespa spp., Dolichovespula spp.). Allergic reactions to the venoms of stinging ants are of major relevance in North and South America (fire ants; Solenopsis spp.), Australia (jumper ant; Myrmecia pilosula) and Asia (Asian needle ant; Pachycondyla chinensis).

In addition to Phospholipase A1 (PLA1) [5], antigen 5 (Ag5) represents one of the most important major venom allergens in almost all allergy-relevant Vespoidea species [6-12]. Only for Myrmecia pilosula, no Ag5 was annotated as allergen so far. Although Ag5 allergens are the most abundant proteins in most Vespoidea venoms, their function within the venoms remains largely unclear [13].

In recent years, the focus in venom allergy research has increasingly shifted from whole venoms to individual allergenic molecules. This had led to the development of component-resolved diagnostics (CRD) $[14 \bullet, 15 \bullet, 16 \bullet$, 17-19], which uses single allergens of the venoms instead of whole venom extracts to measure specific IgE ( $\mathrm{SIgE}$ ) antibodies in patients' sera. Particularly, for the differentiation between cross-reactivity and primary sensitization to HBV and YJV, CRD with species-specific marker allergens such as Ag5 (Ves v 5) and phospholipase A2 (Api m 1) added value. However, due to the high degree of cross-reactivity between the major allergens of vespid venoms, discrimination of allergies to different vespid species such as yellow jackets (Vespula spp.) and paper wasps (Polistes spp.) remains challenging.

Due to their outstanding role as major allergens, $\mathrm{Ag} 5$ proteins build a key element for diagnosis of Vespoidea venom allergy. For instance, molecular diagnosis applying Ag5 (Ves v 5) of yellow jackets has already proven to be able to increase diagnostic sensitivity and has led to the development of advanced diagnostic tests $[20 \bullet \cdot$. Nevertheless, there is an urgent need for new diagnostic concepts, which due to their relevance as allergens surely have to include Ag5 proteins, to dissect primary allergy and cross-reactivity in vespid venom allergy. Moreover, Ag5 proteins, as major sensitizing allergens of Vespoidea venoms, may represent a reliable basis for the design of new therapeutic strategies in Hymenoptera venom allergy.

\section{Antigen 5 Homologs in Different Species and Their Antigenic Cross-reactivity}

Ag5 proteins of Hymenoptera belong to the CAP (cysteinerich secretory proteins, antigen 5 and pathogenesis-related 1 proteins) superfamily, whose members are found in a wide range of organisms including plants as well as members of each of the animal kingdoms and are involved in diverse biological processes such as reproduction, cancer, immune regulation and host defense [13]. The Ag5 proteins form a major and distinct clade of the CAP superfamily and are mainly found in stinging and blood-feeding insects [13]. While most representatives of the CAP superfamily are secreted and function as endocrine or paracrine modulators, the role of Hymenoptera $\mathrm{Ag} 5$ proteins remains elusive [13]. In bloodfeeding ticks, flies and mosquitoes, Ag5 proteins are part of a mixture of salivary proteins that are thought to function either in suppression of the host immune system or in preventing platelet aggregation [21]. This biological function is similar to that found for other CAP proteins, e.g. of parasitic nematodes or lampreys, and, therefore, most likely encoded within the CAP domain (Fig. 1a). The presence of Ag5 allergens (as well as hyaluronidases), which exhibit crossreactivity with their homologues of wasp venom, in the salivary of horseflies and mosquitoes [22], may explain the postulated "wasp-mosquito-horsefly-syndrome", in which wasp venom-allergic patients also experience systemic reactions after bites of mosquitoes or horseflies [23, 24].

Recently, an Ag5-like protein was also identified at transcriptomic level in the venom glands of winter but not of summer bees. However, the recombinantly produced protein showed neither IgG4 reactivity with sera of beekeepers nor cross-reactivity with YJV Ag5 (Ves v 5). This might be explained by a lack of sting exposure in the winter, low abundance in the venom and/or low sequence identity (approx. 25\%) [25].

To date, 26 Vespoidea Ag5 proteins are listed as allergens (Table 1) in the official allergen nomenclature database of the World Health Organization and International Union of Immunological Societies (WHO/IUIS) [26]. An alignment of selected Hymenoptera Ag5 allergens is shown in Fig. 1a. According to the phylogenetic distance between these species (Fig. 2), the Ag5 allergens exhibit a varying degree of sequence identity (Fig. 1b) and, therefore, most likely of crossreactivity.

Ag5 allergens (as well as other allergens) of different Vespula species display a very high degree of sequence homology and are thought to be nearly completely cross-reactive [27] . Although sequence identity between Ag 5 allergens of members of the Vespinae (Vespula spp., Vespa spp., Dolichovespula spp.) is less, they still exhibit pronounced cross-reactivity. The clinical relevance of this cross-reactivity is reflected by the fact that patients primary sensitized to YJV can develop severe, and even fatal, anaphylaxis after a hornet sting and vice versa and, moreover, that hornet-allergic patients can be adequately treated with yellow jacket VIT $[2,28]$. However, one study from Italy (where Vespa crabro extract is available for VIT) suggests that in patients with ascertained primary Vespa allergy, VIT with $V$. crabro venom would be more adequate, at least concerning the safety profile [29]. 


\section{A}

Ves v 5

Ves g 5

Dol m 5

Vesp c 5

Poly p 5

Pol d 5

Pol a 5

Sol i 3

Ves $v 5$

Ves g 5

Dol $m 5$

Vesp c 5

Poly $\mathrm{p} 5$

Pol d 5

Pol a 5

sol i 3

Ves $\mathrm{v} 5$

Ves g 5

Dol m 5

Vesp c 5

Poly p 5

Pold 5

Pol a 5

Sol i 3

Ves $\mathrm{v} 5$

Ves g 5

Dol $m 5$

Vesp c 5

Poly p 5

Pol d 5

Pol a 5

Sol i 3
----NNYCKI-KCLKGGVHTACKYG-SLKP---NCGNKVVVSYGLTKOEKODILKEHNDF 51 ----NNYCKI-KCLKGGVHTACKYE-SLKP---NCANKKVVAYGLTKQEKQDILKEHNDF 51 ----NNYCKI-KCRK-GI HTLCKFGT SMKP---NCGRNVVKAY GLTNDEKNE I LKRHNDF 51 ----NNYCKI-KCRS-GI HTLCKYGT STKP---NCGKNVVKASGLTKQENLEILKQHNEF 51 ----NKYCNI-KCSK-VAHTVCQTGE STKP SSKNCAKVS IT SVGVTEEEKKLIVDEHNRF 54 ----NDYCKI-KCSS-GVHTVCQYGESTKPS-KNCAGKL IKSVGPTEEEKKLIVEEHNRF 53 SSQGVDYCKI-KCPS-GIHTVCQYGESTKPS-KNCAGKVIKSVGPTEEEKKLIVSEHNRF 57 ----VDYCNLESCSQRGS HTMCRYPSSSPS--NAC--RQWSNNGLTEAEKNTIVKRHNEL 52

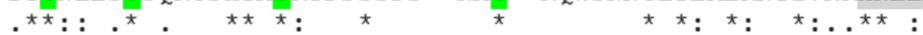

RQKIARGLETRGNPGPQP PAKNMKNLVWNDELAYVAQVWANQCQY-GHDTCRDVAKYQVG 110 RQKIARGLETRGNPGPQP PAKNMKNLVWNDELAY IAQVWANQCQY-GHDTCRDVAKYPVG 110 RQNVAKGLETRGKPGPQP PAKNMNVLVWNDELAK IAQTWANQCDF-NHDDCRNTAKYQVG 110 RQKVARGLETRGNPGPQP PAKSMNTLVWNDELAQIAQVWANQCNY-GHDNCRNSAKYSVG 110 RQKVAQGLETRGN PGPQPAASDMNNLVWNDELAY IAQVWASQCQFFV HDKCRNTAQYQVG 114 RQKVAKGLETRGNPGPQPAASNMNNLVWNDELAKIAQVWASQCQILVHDKCRNTEKYQVG 113 RQKVAQGLE TRGN PGPQPAASDMNDLVWNDELAH IAQVWASQCQFLVHDKCRNTAKYPVG 117 RQRVASGQE SRGN PGPQP PAVSMPALVWDDELAT IAQRWANQCNF-NHDTCRNVDRFAVG 111

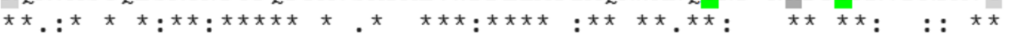

QNVALTGSTAAKYDDPVKLVKMWEDEVKDYNPKKKFSGNDFLKTGHYTQMVWANTKEVGC 170 QNVALTGSTAAKYDNPVKLVKMWEDEVKDYNPKKKFSENNFLKIGHYTQMVWANTKEVGC 170 QNIAISSTTATQF DRPS KLIKQWEDEVTE FNYKVGLQNSNFRKVGHYTQMVWGKT KEIGC 170 ONIAE GSTTADNFGSVSNMVKMWEDEVKDYQY--GSPKNKLNKVGHYTOMVWAKTKEIGC 168 QNIAYSASTA-AY PGVVKLIVLWENEVKDFNYNT GITKENFAKVGHYTQVVWAKT KEVGC 173 QNIAYAGSSN-HFPSVTKLIQLWENEVKDFNYNT GITNKNFGKVGHYTQMVWGNTKEVGC 172 QNIAYAGGSN--L PDVVS LIKLWENEVKDFNYNT GITKQNFAK I GHYTQMVWGKT KEIGC 175 QNIAMTYNS GDNNS PME SFVDMWYDEVDKFDRNKVDYYEFEPSTGHYTQVVWANTKTIGC 171

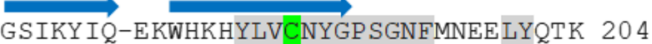
GSIKYIQ-DKWHKHYLVCNYGPSGNFGNEELYQTK 204 GSIKYIE-DNWYT HYLVCNYGPGGNDFNOP IYERK 204 GSIKYIE-NGWHRHYLVCNYGPAGNVGNEPIYERK 202 GSIKYIE-KGMKSHYLVCNYGPAGNVLGAQIYEIK 207 GSLKYVE-KNMQI HYLICNYGPAGNYLGQPIYTKK 206 GSLKYME-NNMONHYLICNYGPAGNYLGQLPYTKK 209 GRIKYKESNGWNANYLVCNYGPSGNYIGQKIYQRK 206

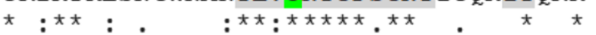

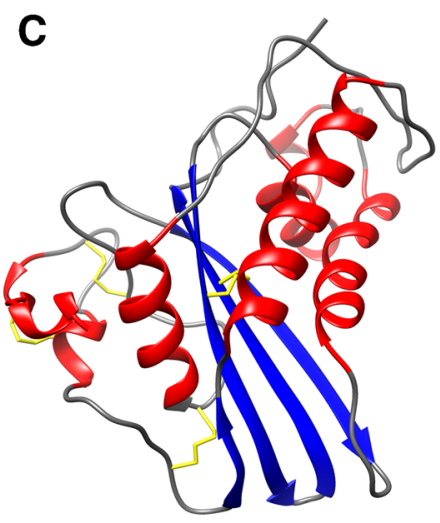

Ves $v 5$

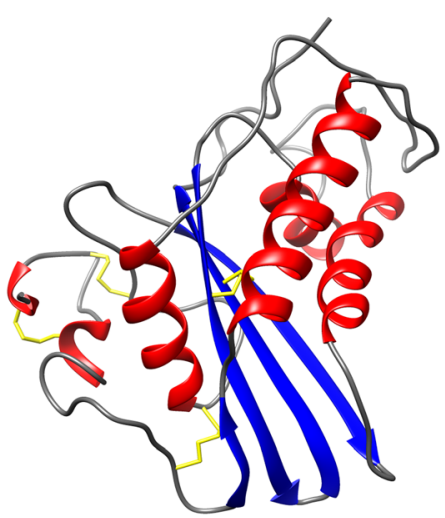

Pol d 5

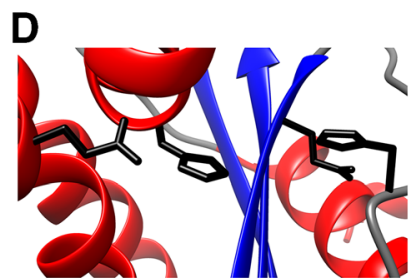

\section{B}

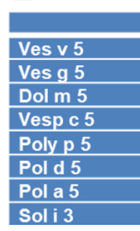

\begin{tabular}{|l|r|}
\hline Ves v 5 & Ves \\
\hline $94 \%$ & 94 \\
\hline $66 \%$ & $66 \%$ \\
\hline $69 \%$ & $69 \%$ \\
\hline $61 \%$ & 61 \\
\hline $60 \%$ & $60 \%$ \\
\hline $59 \%$ & $59 \%$ \\
\hline $50 \%$ & $50 \%$ \\
\hline
\end{tabular}

\begin{tabular}{|c|r|}
\hline Ves a 5 & Dol \\
\hline $94 \%$ & $66 \%$ \\
\hline $66 \%$ & $66 \%$ \\
\hline $69 \%$ & $73 \%$ \\
\hline $61 \%$ & $62 \%$ \\
\hline $60 \%$ & $64 \%$ \\
\hline $59 \%$ & $62 \%$ \\
\hline $50 \%$ & $51 \%$ \\
\hline
\end{tabular}

\begin{tabular}{|c|r|}
\hline Dol m 5 & Vesp \\
\hline $66 \%$ & $69 \%$ \\
\hline $66 \%$ & $69 \%$ \\
\hline $73 \%$ & $73 \%$ \\
\hline $62 \%$ & $63 \%$ \\
\hline $64 \%$ & $61 \%$ \\
\hline $62 \%$ & $63 \%$ \\
\hline $51 \%$ & $54 \%$ \\
\hline
\end{tabular}

\begin{tabular}{|l|r|}
\hline esp c 5 & Poly p \\
\hline $69 \%$ & $61 \%$ \\
\hline $69 \%$ & $61 \%$ \\
\hline $73 \%$ & $62 \%$ \\
\hline & $63 \%$ \\
\hline $63 \%$ & \\
\hline $61 \%$ & $78 \%$ \\
\hline $63 \%$ & $78 \%$ \\
\hline $54 \%$ & $50 \%$ \\
\hline
\end{tabular}

Fig. 1 Antigen 5 homologues and their structure. a Alignment of the mature sequences of selected Hymenoptera antigen 5 allergens. The secondary structure elements identified in Ves v 5 are indicated above the relevant amino acid sequences (red, $\alpha$-helix; blue, $\beta$-strand). These elements are conserved between the different Ag5 proteins. The CAP signature motifs (CAP3, CAP 4, CAP1, CAP2) and the typical motif [ILVP]Y, which is found near the terminus of Ag5 proteins, are marked in light grey. Conserved residues that form the putative active site are marked in dark grey. Cysteine residues that form disulphide bridges are marked in green. Asterisks, colons and periods indicate identical, conserved and semi-conserved residues, respectively. b Percent identity

Cross-reactivity between Ag5 allergens of the Vespinae and Polistinae subfamilies was described to be less pronounced as within the same subfamily [8]. Nevertheless, double-positive sIgE test results to YJV and Polistes dominula venom (PDV) are frequently observed and represent a diagnostic challenge in areas where both species are endemic [30, $31 \bullet \cdot, 32 \bullet \cdot$. Due to a partial cross-reactivity between the Ag5

\begin{tabular}{|l|l|l|}
\hline Pol d 5 & Pol a 5 & Soli 3 \\
\hline $60 \%$ & $59 \%$ & $50 \%$ \\
\hline $60 \%$ & $59 \%$ & $50 \%$ \\
\hline $64 \%$ & $62 \%$ & $51 \%$ \\
\hline $61 \%$ & $63 \%$ & $54 \%$ \\
\hline $78 \%$ & $78 \%$ & $50 \%$ \\
\hline & $86 \%$ & $49 \%$ \\
\hline $86 \%$ & & $50 \%$ \\
\hline $49 \%$ & $50 \%$ & \\
\hline
\end{tabular}

between the different antigen 5 allergens. Sequence identifiers: Ves v 5 (Q05110.1), Ves g 5 (CAJ28930.1), Dol m 5 (P10736.1), Vesp c 5 (P35781.1), Poly p 5 (P86686.1), Pol d 5 (NP_001310265.1), Pol a 5 (Q05109.1), Sol i 3 (XP_011165202.1). c Crystal structure of Ves v 5 (1QNX) [37] and structural model of Pol d 5 [32••]. $\alpha$-helices, $\beta$-strands and coiled regions are shown in red, blue and grey, respectively. Disulphide bridge-forming cysteines are indicated in yellow. d The solvent-exposed cleft (Ves v 5), which contains the putative active site, formed by a conserved dihistidine motif and conserved residues (Glu, Gln) providing a supporting hydrogen bond network

allergens and other venom allergens of European (P. dominula, P. gallicus) and American (e.g. P. annularis, P. exclamans, $P$. fuscatus) Polistes species, which belong to different subgenera (Fig. 2), diagnosis and therapy should be performed with the venoms of endemic species $[30 \bullet, 32 \bullet, 33$, 34]. While in first studies cross-reactivity between red imported fire ant $\mathrm{Ag} 5$ (Sol i3) and vespid Ag5 allergens was 
Table 1 Antigen 5 allergens currently listed in the official WHO/IUIS allergen nomenclature database [26]

\begin{tabular}{|c|c|c|c|}
\hline Species & Common name & Allergen & Sensitization rate ${ }^{\mathrm{a}}$ \\
\hline Dolichovespula arenaria & Yellow hornet & Dol a 5 & $81 \%^{\mathrm{b}}$ \\
\hline Dolichovespula maculata & White-faced hornet & Dol m 5 & $65 \%[7]$ \\
\hline Pachycondyla chinensis & Asian needle ant & Pac c 3 & $83 \%[36]$ \\
\hline Polistes annularis & American paper wasp & Pol a 5 & $44-65 \%[30 \bullet, 32 \bullet \bullet]^{2}$ \\
\hline Polistes dominula & European paper wasp & Pol d 5 & $72 \%[30 \bullet]$ \\
\hline Polistes exclamans & American paper wasp & Pol e 5 & $90 \%[7]$ \\
\hline Polistes fuscatus & Golden/Northern paper wasp & Pol f 5 & $69 \%[83]$ \\
\hline Polistes gallicus & European paper wasp & Pol g 5 & $80-100 \%[84]$ \\
\hline Polistes metricus & Metricus paper wasp & Pol m 5 & Yes $[83]^{\mathrm{c}}$ \\
\hline Polybia paulista & Polybia wasp & Poly p 5 & $100 \%[57 \bullet]$ \\
\hline Polybia scutellaris & Polybia wasp & Poly s 5 & $58-70 \%[32 \bullet \bullet]^{\mathrm{c}}$ \\
\hline Solenopsis geminata & Tropical fire ant & Sol g 3 & $100 \%[85]$ \\
\hline Solenopsis invicta & Red imported fire ant & Sol i 3 & $67 \%[9]$ \\
\hline Solenopsis richteri & Black fire ant & Sol r 3 & Yes $[86]^{c}$ \\
\hline Solenopsis saevissima & Brazilian fire ant & Sol s 3 & $?$ \\
\hline Vespa crabro & European hornet & Vesp c 5 & $67 \%[32 \bullet]^{\mathrm{c}}$ Yes $[87]$ \\
\hline Vespa magnifica & Hornet & Vesp ma 5 & $73-91 \%^{\mathrm{b}}$ \\
\hline Vespa mandarinia & Giant Asian hornet & Vesp m 5 & $?$ \\
\hline Vespa velutina & Asian hornet & Vesp v 5 & $86 \%^{\mathrm{b}}$ \\
\hline Vespula flavopilosa & Downy yellow jacket & Ves f 5 & $?$ \\
\hline Vespula germanica & German yellow jacket & Ves g 5 & Yes [88] \\
\hline Vespula maculifrons & Eastern yellow jacket & Ves $\mathrm{m} 5$ & Yes [10] \\
\hline Vespula pensylvanica & Western yellow jacket & Ves p 5 & Yes [89] \\
\hline Vespula squamosa & Southern yellow jacket & Ves s 5 & $79 \%[7]$ \\
\hline Vespula vidua & Long/Widow yellow jacket & Ves vi 5 & $?$ \\
\hline Vespula vulgaris & Common yellow jacket & Ves v 5 & $85-100 \%$ (Table 2) \\
\hline
\end{tabular}

A question mark indicates that no data was found. Yes means that sensitization was shown but data about sensitization rates is not given.

${ }^{a}$ Determined by different methods and with highly variable patient numbers

${ }^{\mathrm{b}}$ Data obtained from the WHO/IUIS allergen nomenclature database

${ }^{\mathrm{c}}$ Analyzed patient cohort is not entirely suitable to assess sensitization to the given allergen described to be absent $[8,35]$, a later study demonstrated pronounced cross-reactivity [32••]. Accordingly, crossreactivity between Ag5 of the Asian needle ant (Pac c 3) and YJV Ag5 (Ves v 5) was demonstrated [36]. The clinical relevance of this cross-reactivity is so far unclear.

In recent study, which applied recombinantly produced Ag5 allergens from seven allergy-relevant species (Vespula vulgaris, Vespa crabro, Dolichovespula maculata, Polistes dominula, Polistes annularis, Polybia scutellaris and Solenopsis invicta), pronounced SIgE cross-reactivity between all Ag5 allergens was demonstrated in cohorts of primary YJV- and PDV-sensitized patients [32••]. Moreover, robust effector cell activation of YJV-allergic patients by all Ag5 allergens was demonstrated in basophil activation test (BAT). The clinical relevance of this observation at a symptom-based level remains elusive, as most of the patients were only stung by one of the species.

\section{Structural Aspects of Hymenoptera Antigens 5}

So far, the crystal structures of YJV Ag5 Ves v 5 and fire ant Ag5 Sol i 3 were solved [37, 38]. The secondary structure elements (Fig. 1a) are arranged in an $\alpha-\beta$ - $\alpha$-sandwich fold consisting of a central antiparallel $\beta$-sheet surrounded on both sides by $\alpha$-helices (Fig. 1c). The alignment in Fig. 1a shows that most of the structural elements in Ves v 5 can be expected in all Hymenoptera Ag5 proteins, resulting in an identical fold that is characteristic for all members of the CAP superfamily [13]. Hence, structural modeling of Hymenoptera Ag5 proteins results in very similar three-dimensional structures [32••], as depicted exemplarily for PDV Ag5 Pol d 5 in Fig. 1c. These similar structures with conserved surface areas but also differences in side-chain properties of exposed amino acids suggest the presence of conserved but also unique B cell 


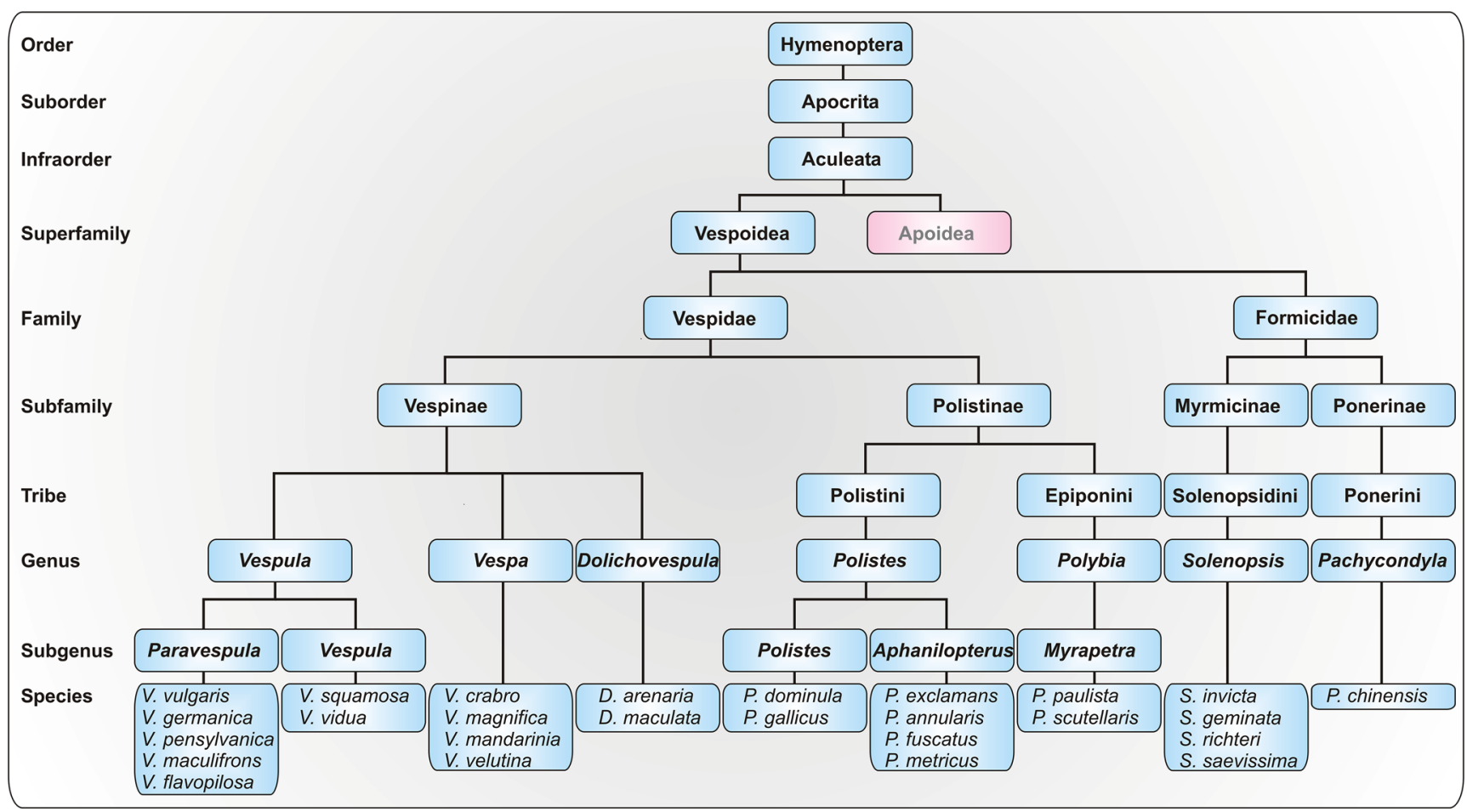

Fig. 2 Taxonomy of Hymenoptera species for which an antigen 5 is annotated as allergen. Other allergy-relevant Hymenoptera species such as honeybees and bumblebees belong to the superfamily Apoidea

epitopes [32••, 37, 38], explaining the different pattern of cross-reactivity between closely related and phylogenetically more distant members of the Ag5 family. The Ag5 structures are stabilized by a number of disulphide bridges (Fig. $1 \mathrm{a}$ and c), which provide the thermal, $\mathrm{pH}$ and proteolytic stability of CAP proteins [13].

The fact that the $\mathrm{Ag} 5$ proteins, in contrast to other members of the CAP superfamily, consist only of the CAP domain, which is characterized by 4 consensus sequences (CAP1-4) (Fig. 1a), implies that their function must be encoded within this domain [13]. Of note, 4 conserved amino acids, including 2 histidines (Fig. 1a), are solvent exposed and located in an elongated cleft (Fig. 1d). These residues are able to provide a hydrogen bond network and are believed to form a putative active site, perhaps with a bound divalent cation $[13,37]$.

\section{IgE Sensitization to Antigens 5}

The rates of sIgE sensitization to Ag5 proteins that are annotated as allergens are depicted in Table 1. Of note, the sensitization rates to the different $\mathrm{Ag} 5$ allergens have been analyzed using a variety of methods, including immunoblotting, ELISA, effector cell activation tests and commercially available sIgE assay platforms. Moreover, inclusion criteria of patients as well as analyzed patient numbers, ranging from a few to hundreds, highly differ in the studies. Therefore, the obtained $\operatorname{sIgE}$ sensitization rates to the different $\mathrm{Ag} 5$ allergens are difficult to compare and a comprehensive picture of Ag5 sensitization in patients with primary allergy to the respective species cannot be drawn with absolute certainty. Nevertheless, the available studies suggest that Ag5 proteins most likely represent the most potent major allergens in all allergy-eliciting Vespoidea species, for which an allergenic Ag5 was identified.

Studies, addressing sensitization rates in large, welldefined patient populations on $\operatorname{sg} \mathrm{E}$ assay platforms, which are more standardized and more applicable for measurements in larger study cohorts, are currently only available for Ves v 5 from YJV. In a first study that used a no longer available liquid-phase detection system (ADVIA Centaur), sIgE to Ves $\mathrm{V} 5$ was detected in 89/100 (89\%) of patients with a history of YJV allergy and positive skin test to YJV. When the inclusion criteria were extended to patients with sIgE to YJV, 87/91 (96\%) were positive to Ves v 5 [39]. In a following study using the ImmunoCAPTM system (Thermo Fisher Scientific, Uppsala, Sweden), Ves v 5-sIgE was found in 53/ 59 (90\%) of patients with history of YJV allergy. Including only patients with detectable sIgE to YJV raised the sensitization rate to $94 \%$ (48/51), whereby no differences between YJV-monosensitized (ms) (94\%) and YJV/HBV-double-sensitized (ds) (95\%) patients were observed [40]. A follow-up study by Köhler et al. found sIgE to Ves v 5 in 158/170 (93\%) of YJV-allergic patients with sIgE to YJV (92\% and 94\% in $\mathrm{ms}$ and ds patients, respectively) [41]. Using the same assay platform, Schiener et al. detected SIgE to Ves v 5 in $42 / 43$ 
$(98 \%)$ [32••] and Ebo et al. in 131/148 (89\%) of patients with a history of YJV allergy; $90 \%$ (82/91) in YJV-ms patients and $92 \%$ (43/47) in patients ds to YJV and HBV or with discrepant YJV-sIgE and skin test results [42]. Comparably, in a large cohort of patients with YJV-allergy (including ms and ds patients), 277/308 (90\%) were reactive to Ves v 5 [20••], whereas in another cohort of YJV-ms patients, the value was slightly lower with $85 \%(169 / 200)$ [43].

Interestingly, Selb et al. measured sensitization to Ves v 5 in the same patient population of YJV-ms patients using the ImmunoCAPTM and the Immulite ${ }^{\mathrm{TM}}$ (Siemens Healthcare Diagnostics, Eschborn, Germany) system and found sensitization rates of $82 \%(90 / 110)$ and $93 \%(102 / 110)$, respectively [44]. In another study, investigating 111 patients with YJV allergy, these results were confirmed. Here, the sensitization rates to Ves v 5 were $87 \%$ (96/111) and 92\% (102/111) using the ImmunoCAPTM and the Immulite ${ }^{\mathrm{TM}}$ system, respectively [45]. However, the same study demonstrated a lower specificity for sIgE detection to Ves v 5 on the Immulite ${ }^{\mathrm{TM}}$ system (92\% compared to $100 \%$ for the ImmunoCAPTM system). These differences in obtained sensitization rates, using the two sIgE assay systems mentioned above, are most likely not due to the quality of allergens used but rather to the difference in calibration approaches, resulting in an overestimation of sIgE levels in one system [46••, 47, 48]. An overview of sIgE sensitization rates to Ves $\mathrm{v} 5$ in different study populations is given in Table 2 in the column "Sensitivity Ves v 5".

\section{Antigens 5 in Routine Allergy Diagnosis and Their Diagnostic Sensitivity}

Routine diagnosis of Hymenoptera venom allergy is based on a combination of clinical history of a systemic sting reaction and the proof of sensitization by skin testing and/or in vitro measurement of venom-specific IgE antibodies. In recent years, CRD of Hymenoptera venom allergy rapidly evolved $[14 \bullet, 15 \bullet, 16 \bullet, 17-19]$. In contrast to extract-based sIgE diagnosis that measures $\mathrm{sIgE}$ levels to native whole venom extracts, levels of sIgE to single allergens of the venoms are determined in CRD. Thus, CRD not only provides information on whether a patient has SIgE to the whole venom, but also which allergens of the venoms are relevant for sensitization. Sensitization profiles obtained in this way can help to discriminate between cross-reactivity and primary sensitization to different venoms. This particularly holds true for vespid venom and HBV allergy since marker allergens, specific for the respective venoms, exist. Additionally, allergens for CRD can be recombinantly produced without cross-reactive carbohydrate determinants (CCDs). Hence, in contrast to venom extract-based diagnosis, clinically irrelevant sensitization to $\mathrm{CCDs}$ is excluded in $\mathrm{CRD}$ with recombinant $\mathrm{CCD}$-free allergens $\left[15 \bullet, 16^{\bullet}\right.$. So far, only the Ag5 allergens of YJV
(Ves v 5) and PDV (Pol d 5) are available for routine CRD on various diagnostic platforms for either singleplex (Ves v 5: Thermo Fisher Scientific, Siemens Healthcare Diagnostics and Dr. Fooke Laboratories; Pol d 5: Thermo Fisher Scientific) or multiplex testing (Ves v 5 and Pol d 5: Euroimmun and Macro Array Diagnostics).

Conclusive data about the diagnostic sensitivity is currently available for Ves v 5 only. Using Ves v 5 alone for the diagnosis of patients with a history of YJV allergy, the diagnostic sensitivity ranges between $89 \%$ and $92 \%$ using the currently available diagnostic assay systems [20••, 40, 42, 45, 49, 50]. In patient cohorts, for which detectable sIgE to YJV was inclusion criterion, diagnostic sensitivity ranged between 82 and $98 \%$ [32••, 40-44]. Interestingly, the lowest values were found in patient cohorts monosensitized to YJV. This phenomenon might be explained by lower levels of sIgE to individual allergens in monosensitized patients, an effect that was also found in HBV-allergic patients and that might be explained by a more advanced state of allergic immune deviation in double-sensitized subjects [41]. As outlined before, for patient cohorts that were analyzed using the ImmunoCAPTM and the Immulite ${ }^{\mathrm{TM}}$ system, higher diagnostic sensitivity (but lower specificity) was obtained with the Immulite ${ }^{\mathrm{TM}}$ system. An overview about diagnostic sensitivity using Ves v 5 for diagnostic work-up in YJV-allergic patients is given in Table 2. The sensitivity of Pol d 5 for the diagnosis of PDV allergy is hard to assess, as a high percentage of the respective patient populations is double-sensitized to PDV and YJV with unknown primary sensitizer.

PLA1 (Ves v 1) was the second YJV major allergen that was introduced for routine diagnosis of YJV allergy (ImmunoCAPTM system). The use of the combination of both major allergens resulted in a sensitivity of $92 \%$ $100 \%$ for the diagnosis of YJV allergy [20••, 42-45, 49-51]. Hence, the addition of Ves v 1 to Ves v 5 increased diagnostic sensitivity by an additional $4 \%$ to $11 \%$ in the different study populations (Table 2). However, a small percentage of YJV-allergic patients cannot be diagnosed using the commercially available YJV allergens. So far, it remains elusive if this diagnostic gap can be filled by other YJV allergens, such as Ves $\mathrm{v} 2$ or Ves v 3.

It is important to note that the levels of sIgE to whole venom extracts or to individual venom allergens do not correlate with the severity of the sting reaction [43, 44, 52•]. Although no correlation between the number of recognized allergens of a venom and the severity of the sting reaction can be observed in clinical routine, detailed studies addressing this are still missing.

Interestingly, a study that addressed sensitization profiles of YJV-allergic patients with mastocytosis and/or elevated basal serum tryptase found sensitization to Ves v 5 in $42 / 49(86 \%)$ of patients and the addition of Ves $\mathrm{V} 1$ increased diagnostic sensitivity to $92 \%(45 / 49)$ using the 
Table 2 Diagnostic sensitivity of Ves $\mathrm{v} 5$ and Ves $\mathrm{v} 1$ in diagnosis of patients with a history of yellow jacket venom allergy

\begin{tabular}{|c|c|c|c|c|c|c|}
\hline Patients & $\begin{array}{l}\text { Sensitivity } \\
\text { YJV }\end{array}$ & $\begin{array}{l}\text { Sensitivity } \\
\text { Ves v } 5\end{array}$ & $\begin{array}{l}\text { Sensitivity Ves } \\
\text { v } 5 / \text { Ves v } 1\end{array}$ & Method $^{\mathrm{a}}$ & Remarks & Reference \\
\hline $\begin{array}{l}100 \\
91\end{array}$ & $\begin{array}{l}91 \%(91)^{\mathrm{b}} \\
\mathrm{IC} \operatorname{sIgE}\end{array}$ & $\begin{array}{l}89 \%(89) \\
96 \%(87)\end{array}$ & $\begin{array}{l}\text { n.d. } \\
\text { n.d. }\end{array}$ & ADVIA Centaur ${ }^{\mathrm{d}}$ & & [39] \\
\hline $\begin{array}{l}59 \\
32\end{array}$ & $\begin{array}{l}86 \%(51)^{\mathrm{b}} \\
\mathrm{IC} \operatorname{sIgE}\end{array}$ & $\begin{array}{l}90 \%(53) \\
94 \%(30)\end{array}$ & $\begin{array}{l}\text { n.d. } \\
\text { n.d. }\end{array}$ & ImmunoCAP & $\mathrm{ms}$ & {$[40]$} \\
\hline 19 & IC sIgE & $95 \%(18)$ & n.d. & & ds & \\
\hline 8 & IC neg. & $63 \%(5)$ & n.d. & & & \\
\hline 22 & IC $\operatorname{sIgE}$ & n.s. & $100 \%(22)$ & ImmunoCAP & $\mathrm{ms}$ & {$[51]$} \\
\hline 200 & IC sIgE & $85 \%(169)$ & $92 \%(184)$ & ImmunoCAP & $\mathrm{ms}$ & {$[43]$} \\
\hline $\begin{array}{l}163 \\
26\end{array}$ & $\begin{array}{l}\text { n.s. } \\
\text { IC neg. }\end{array}$ & $\begin{array}{l}92 \%(150) \\
65 \%(17)\end{array}$ & $\begin{array}{l}96 \%(156) \\
65 \%(17)\end{array}$ & ImmunoCAP & & {$[50]$} \\
\hline 308 & $\begin{array}{l}83 \% \\
\quad(257)^{b}\end{array}$ & $90 \%$ (277) & $96 \%(296)$ & ImmunoCAP & & {$[20 \bullet \bullet]$} \\
\hline 308 & $\begin{array}{l}97 \% \\
\quad(298)^{\mathrm{c}}\end{array}$ & $90 \%(277)$ & $96 \%(296)$ & & & \\
\hline 148 & $\begin{array}{l}89 \% \\
\quad(131)^{\mathrm{b}}\end{array}$ & $89 \%(131)$ & $94 \%$ (139) & ImmunoCAP & & {$[42]$} \\
\hline 91 & IC sIgE & $90 \%(82)$ & $98 \%(89)$ & & $\mathrm{ms}$ & \\
\hline 17 & IC neg. ${ }^{b}$ & $71 \%(12)$ & $71 \%(12)$ & & & \\
\hline $\begin{array}{l}170 \\
103\end{array}$ & $\begin{array}{l}\text { IC sIgE } \\
\text { IC sIgE }\end{array}$ & $\begin{array}{l}93 \%(158) \\
92 \%(95)\end{array}$ & $\begin{array}{l}\text { n.d. } \\
\text { n.d. }\end{array}$ & ImmunoCAP & $\mathrm{ms}$ & {$[41]$} \\
\hline 67 & IC $\operatorname{sIgE}$ & $94 \%(63)$ & n.d. & & ds & \\
\hline 43 & IC sIgE & $98 \%(42)$ & n.d. & ImmunoCAP & & {$[32 \bullet \bullet]$} \\
\hline 111 & $\begin{array}{l}100 \% \\
(111)\end{array}$ & $87 \%(96)$ & $98 \%$ (109) & ImmunoCAP & & {$[45]$} \\
\hline 111 & $98 \%(109)$ & $92 \%(102)$ & n.d. & Immulite & & \\
\hline $\begin{array}{l}110 \\
110\end{array}$ & $\begin{array}{l}\text { IC sIgE } \\
\text { IC sIgE }\end{array}$ & $\begin{array}{l}82 \%(90) \\
93 \%(102)\end{array}$ & $\begin{array}{l}90 \%(99) \\
97 \%(107)\end{array}$ & $\begin{array}{l}\text { ImmunoCAP } \\
\text { Immulite/ImmunoCAP }\end{array}$ & $\begin{array}{l}\mathrm{ms} \\
\mathrm{ms}\end{array}$ & {$[44]$} \\
\hline $\begin{array}{l}25 \\
49^{\mathrm{e}}\end{array}$ & $\begin{array}{l}92 \%(23)^{\mathrm{b}} \\
88 \%(43)^{\mathrm{b}}\end{array}$ & $\begin{array}{l}92 \%(23) \\
86 \%(42)\end{array}$ & $\begin{array}{l}100 \%(25) \\
92 \%(45)\end{array}$ & Immulite & & [49] \\
\hline
\end{tabular}

n.d. not determined, n.s. not shown, IC sIgE $\operatorname{sIgE}$ to $\mathrm{YJV} \geq 0.35 \mathrm{kU} \mathrm{A}_{\mathrm{A}} / \mathrm{L}$ was inclusion criterion, IC neg. $\operatorname{sIgE}$ to $\mathrm{YJV}<0.35 \mathrm{kU} \mathrm{A} / \mathrm{L}$ was inclusion criterion, $m s$ yellow jacket venom-monosensitized, $d s$ yellow jacket venom and honeybee venom-double-sensitized

${ }^{\text {a }}$ Values $\geq 0.35 \mathrm{kU} / \mathrm{L}$ were considered positive

${ }^{\mathrm{b}}$ YJV extract not spiked with Ves $\mathrm{v} 5$ was used for measurement

${ }^{\mathrm{c}}$ YJV extract spiked with Ves v 5 was used for measurement

${ }^{\mathrm{d}}$ No longer available

${ }^{\mathrm{e}}$ Patients with mastocytosis and/or elevated baseline serum tryptase
Immulite ${ }^{\mathrm{TM}}$ system (research prototype allergen assays) [49]. In this high-risk patient group, diagnostic sensitivity could be increased to $100 \%$ only by Ves v 1 - and Ves v 5based CRD and lowering the threshold to $0.1 \mathrm{kU}_{\mathrm{A}} / \mathrm{L}$. For two of the patients with severe anaphylaxis, who exhibited negative intracutaneous skin tests and YJV-sIgE $<0.1 \mathrm{kU} /$ L, this was the only way to verify sensitization [49]. Other authors confirmed an improved diagnostic sensitivity in YJV-allergic patients with mastocytosis by lowering the threshold to $0.17 \mathrm{kU} / \mathrm{L}$, while good specificity was retained [53]. It was demonstrated before that sIgE levels between 0.1 and $0.35 \mathrm{kU} / \mathrm{L}$ can be measured with high accuracy on the major singleplex sIgE immunoassay platforms and should be considered in the context of a clear clinical history of venom allergy, irrespective of the presence of mast cell disorders [54, 55].

Clear gaps exist for accurate diagnosis of allergy to neotropical wasps in South America. For instance, Polybia paulista, a species that is common in the southeast of Brazil, represents a neglected health problem and causes a large number of severe systemic and even fatal allergic reactions [56]. Here, no routine diagnostics is available so far, a fact, leading to challenges in identification of primary sensitization. Recently, also Ag5 (Poly p 5) and PLA1 (Poly p 1) were identified as targets of interest for diagnosis of Polybia venom allergy $[57 \cdot, 58]$. 


\section{Antigen 5-Spiked Venom Extracts for Diagnosis}

When Ves $\mathrm{v} 5$ became available for routine diagnosis, different studies demonstrated that SIgE to this allergen could be detected in $63 \%$ to $71 \%$ of patients with a history of YJV allergy but negative sIgE test results with YJV (Table 2) [40, $42,50]$. Following these observations, Vos et al. demonstrated in a population of 308 patients with confirmed YJV allergy that only $83 \%$ could be diagnosed with the conventional YJV ImmunoCAPTM, while sensitization could be verified in $96 \%$ using the individual allergens Ves v 1 and Ves v 5 [20••]. Of the extract-negative patients, only one was tested positive for Ves $\mathrm{v} 1$, whereas $84 \%(42 / 51)$ were positive for Ves $\mathrm{v} 5$. Moreover, and in contrast to Ves $\mathrm{v} 1$, in the extract-positive patients, the levels of SIgE to Ves $v 5$ were substantially higher than to YJV extract. These results suggested a shortage of reactive Ves $\vee 5 \mathrm{IgE}$ epitopes in the diagnostic extract. As Ves $\mathrm{v} 5$ is the most abundant protein in YJV, it can only be speculated about the reasons for this reduced immunoreactivity. Presuming that an underrepresentation in the venom extract is unlikely, possible explanations could be an inefficient coupling to the solid phase of the assay or a masking of IgE epitopes by natural ligands in the venom extract.

In the same study, SIgE reactivity of the patient cohort with a Ves v 5-spiked YJV ImmunoCAPTM was analyzed. Compared with the conventional, the Ves $\vee 5$-spiked ImmunoCAPTM yielded substantially higher sIgE values in Ves v 5-positive sera and diagnostic sensitivity increased from 83 to $97 \%$ (Table 2). No relevant differences in reactivity were observed in Ves v 5-negative sera. The increase in sensitivity was not accompanied by a change in specificity as demonstrated using sera of $51 \mathrm{HBV}$-allergic patients. In 18/19 skin test-negative YJV-allergic patients, sensitization could be verified using the Ves v 5-spiked YJV ImmunoCAPTM. Together, the combination of skin tests and $\operatorname{sgE}$ detection to Ves $\mathrm{v} 5$ spiked YJV confirmed sensitization in 300/301 patients $[20 \bullet$ ]. Consequently, the new Ves v 5-spiked YJV ImmunoCAPTM was introduced in summer 2012.

The usefulness of the new test was then also demonstrated in a small study, in which 11 Ves $v$ 5-reactive patients who were negative to the conventional test could be diagnosed using the new Ves v 5-spiked YJV immunoassay [42]. Moreover, a French multicenter study was performed on Ves V 5- and Pol d 5-spiked YJV and PDV ImmunoCAPs ${ }^{\mathrm{TM}}$, respectively [59]. Here, it was also demonstrated that the use of the Ag5-spiked venom extracts results in an improved diagnostic sensitivity, but also in higher numbers of double-positive test results. Moreover, it was shown that the measurement of $\operatorname{sIgE}$ to Ves $v 5$, Pol d 5 and Ves $\mathrm{v} 1$ in sera without detectable sIgE to the spiked extracts results only in minimal diagnostic sensitivity improvements. The improved sensitivity of both, Ag5-spiked YJV and PDV
ImmunoCAPs ${ }^{\mathrm{TM}}$, was also confirmed for a population of Japanese venom-allergic patients [60]. In contrast to the previous reports, one study found only a slight, not significant increase of sensitivity from $94 \%$ (106/113) to $96 \%$ (87/91) using the conventional and Ves $v 5$-spiked extract, respectively [61]. Moreover, a comparable decrease of specificity of both tests was observed with increasing levels of total IgE.

\section{Antigen 5 for the Discrimination Between YJV and HBV Allergy}

Double-positive sIgE test results to YJV and HBV are frequently observed [39, 40, 51, 62]. These double-positive results may either reflect true primary sensitization to both venoms or may be caused by IgE directed against CCDs, which are present on most natural Hymenoptera venom allergens $[63,64]$ or to homologous allergens present in both venoms. In the first case, VIT with both venoms is recommended, while in the second scenario, VIT with the primary sensitizing venom is sufficient. As venom extract-based $\operatorname{sgE}$ testing does not allow discrimination between cross-reactivity and primary sensitization to both venoms, double-positive results strongly hamper the choice of the correct venom for VIT or might even lead to unnecessary treatment with both venoms, particularly in patients who were not able to correctly identify the culprit insect.

Fortunately, molecular or component-resolved diagnostics with recombinantly produced, CCD-free species-specific marker allergens, which are present in either YJV (Ves v 1, Ves v 5) or HBV (Api m 1, Api m 3, Api m 4, Api m 10), has proven to be able to unequivocally identify primary sensitization to a given venom in many cases $[14 \cdot, 15 \bullet, 16 \bullet, 17,18]$. Other allergens such as the hyaluronidases (Ves v 2 and Api $m$ 2) or dipeptidyl peptidases (Ves $\vee 3$ and Api m 5) share similarities and, thus, exhibit a varying degree of cross-reactivity.

In a first study using Ves $\mathrm{v} 5$ and Api $\mathrm{m} 1$ on the no longer available ADVIA Centaur platform, reactivity to both allergens and, hence, primary allergy to both venoms, was confirmed in 34/63 (54\%) of venom extract-double-positive patients, while in the others, reactivity to only one of the marker allergens was detected [39]. A following study measured $\operatorname{sigE}$ to Ves $\mathrm{v} 5$ and Api $\mathrm{m} 1$ on the ImmunoCAPTM platform and confirmed that sIgE detection to both marker allergens allows reliable discrimination between primary allergy and crossreactivity in patients double-sensitized to venom extracts [40]. For instance, primary sensitization to both allergens was found in only $24 \%$ (8/33) of CCD-negative patients with double-positive tests to venom extracts. Moreover, enhanced diagnostic utility of both marker allergens was also demonstrated for the Immulite ${ }^{\mathrm{TM}}$ platform [44]. Ves $\mathrm{v} 1$ was the second YJV allergen that was introduced for CRD. It was found that sensitivity of the two YJV allergens is sufficient 
and that a positive sIgE test result with one of them is indicative for YJV VIT in patients who are double-positive to both venoms and for whom the culprit insect could not be identified [42, 51]. However, missing reactivity to Api $\mathrm{m} 1$ does not necessarily exclude primary HBV allergy. As the detection of sIgE to this HBV major allergen is not always sufficient to distinguish YJV and HBV allergy [65], additional allergens of HBV (Api m 2, Api m 3, Api m 5, Api m 10) were introduced (ImmunoCAPTM) that further increased diagnostic sensitivity of CRD [41]. Interestingly, Gattinger et al. demonstrated that the panel of Ves v 1, Ves v 5, Api m 1 and Api m 10 allowed the identification of the culprit venom in $98 \%(85 / 87)$ of patients sensitized to YJV and/or HBV with good agreement to skin testing [52•]. Contrary, another study questioned the ability of the available allergen panel to resolve doublesensitization, as $70 \%$ (69/98) of the patients double-sensitized to venom extracts were also double-sensitized with at least one allergen of YJV and HBV. A possible explanation was found in the unavailability of potentially cross-reactive allergens from both venoms for CRD [66••]. However, it is not clear to which extend this phenomenon might be caused by true primary sensitization to both venoms (47\% and $13 \%$ of patients showed also double-positive and double-negative skin tests, respectively).

Although diagnostic sensitivity of the currently available allergen panel, particularly of HBV, is not $100 \%$, CRD has clearly improved discrimination of primary allergy and crossreactivity in YJV and HBV allergy and, hence, facilitated correct prescription of VIT.

\section{Antigens 5 for the Discrimination Between YJV and PDV Allergy}

In Southern Europe, double sensitization to YJV and PDV is more frequently observed than that to vespid venom and HBV [34, 67, 68]. Here, a definite resolution of cross-reactivity and true primary allergy to both venoms is rarely possible due to a high degree of cross-reactivity between the major allergens of the venoms. Certainly, cross-reactivity between the antigens 5 (Ves v 5 and Pol d 5) as most relevant major allergens is of considerable importance. This was demonstrated by the fact that $17 / 20(85 \%)$ of YJV-allergic patients from Germany, where PDV allergy is virtually not present, had also sIgE to Pol d 5 in ImmunoCAPTM measurements. Vice versa, 12/16 (75\%) Pol d 5-reactive PDV-allergic patients from Spain (a concomitant YJV allergy cannot be fully excluded) were also reactive to Ves v 5 [32••].

Monsalve et al. demonstrated that comparing the levels of sIgE to the Ag5 allergens (Ves v 5 and Pol d 5) and phospholipases A1 (Ves v 1 and Pol d 1) allows a reliable identification of the culprit venom in $67 \%$ of double-sensitized allergic patients [30•]. A subsequent study of a very small patient cohort showed that the detection of sIgE against the same four allergens could determine the correct venom for immunotherapy in the majority, but not in all patients [69]. However, only Pol d 5 is currently available for routine diagnosis of PDV allergy on the most common sIgE singleplex platform.

To date, the gold standard to resolve double sensitization in PDV and YJV allergy is CAP-inhibition assays with PDV and YJV [31••, 70, 71]. Current limitations of the commercially available homologous allergens Pol d 5 and Ves v5 to distinguish between YJV and PDV allergy in double-positive patients by CRD were demonstrated by the fact that a good accordance between Ag5-based CRD and CAP-inhibition assays can only be achieved when the value of $\operatorname{sigE}$ in $\mathrm{kU}_{\mathrm{A}} / \mathrm{L}$ to Ves $\mathrm{v} 5$ is about twice of those to Pol d 5 and vice versa [71, 72]. However, a later multicenter study did not find any agreement between CAPinhibition test results and double sIgE values of Ves v 5 over Pol d 5 or vice versa [31••].

The available data demonstrates that the use of Ag5 allergens in CRD has extensive limitations in discriminating double-positivity in PDV and YJV allergy. Hence, the commercial availability of additional cross-reactive major allergen pairs (at least an addition of Pol d 1) for routine diagnosis might be beneficial for uncovering primary sensitization in PDV and YJV double-sensitized patients.

Due to the increasing spread of Polistes dominula on several continents [73-77], associated diagnostic problems are likely to gain importance in other areas of the world.

\section{Conclusions}

Ag5 proteins represent a family of very potent allergens that are of major relevance in allergies to venoms of members of the Vespoidea superfamily. Although the function of Ag5 proteins in venoms remains unsolved, the study of these proteins with considerable allergenic potency may help to elucidate the molecular and immunological basis of allergenicity. Moreover, Ag5 allergens have proven to be indispensable for accurate diagnosis of venom allergy and build an essential key element for the discrimination of primary allergy and cross-reactivity in doublepositivity to honeybee and vespid venom in CRD. However, the available data demonstrates that Ag5based testing is insufficient for the differentiation between allergies to different vespid species. In this field, novel approaches, including additional allergens for CRD, are urgently needed for adequate diagnosis. Furthermore, Ag5 allergens may help to fill current diagnostic gaps such as proper diagnosis of Polybia venom allergy in South America [78•]. Moreover, the use of single allergens in BAT in the future might be helpful in the investigation of double-sensitized patients or in patients with a 
clear history of venom allergy but negative $\operatorname{sg} E$ and skin tests [79].

Some studies already identified major $\mathrm{T}$ and $\mathrm{B}$ cell epitopes of Ag5 allergens or generated hypoallergenic folding variants with the aim to design novel therapeutic strategies [80-82]. However, the outstanding efficacy of the currently available VIT for Vespoidea venom allergy will make it difficult for such strategies to be realized. Nevertheless, Ag5-based therapeutics might be a practicable way for allergies to species, for which VIT is not available so far or for which substantial amounts of venom are difficult to obtain.

Authors' Contributions All authors contributed to the conception and writing of the manuscript as well as read and approved the final manuscript.

Funding Information Open Access funding provided by Projekt DEAL. This work was supported by the Helmholtz Association, Future Topic "Immunology and Inflammation" (ZT-0027) to SB and CBS-W and in part by a grant (NR66-0004) of the von-Behring-Röntgen-Stiftung, Marburg and UKGM Research Funding 7/2017GI, Giessen, to TJ. Moreover, São Paulo Research Foundation (FAPESP) provided financial support (Grants \#2019/02298-3 and 2017/07988-2.) to MLB.

\section{Compliance with Ethical Standards}

Conflict of Interest Dr. Blank reports non-financial support from ALK-Abelló, grants and personal fees from Bencard Allergie $\mathrm{GmbH}$, personal fees from Teomed $\mathrm{AG}$, grants and personal fees from Thermo Fisher Scientific, grants from Allergy Therapeutics, outside the submitted work. Dr. Schmidt-Weber reports grants and personal fees from Bencard, grants from Leti Pharma, grants and personal fees from Allergopharma, grants and personal fees from PLS Design, outside the submitted work. In addition, Dr. Schmidt-Weber has a patent Patent on dignostic success prediction in AIT pending. Dr. Jakob reports grants, personal fees and non-financial support from ALK-Abelló, personal fees and nonfinancial support from Bencard/Allergy Therapeutics, grants, personal fees and non-financial support from Novartis, personal fees and non-financial support from Thermo Fisher Scientific, personal fees from Celgene, personal fees from Allergopharma, outside the submitted work. The other authors declare no conflict of interest.

Human and Animal Rights This article does not contain any studies with human or animal subjects performed by any of the authors.

Open Access This article is licensed under a Creative Commons Attribution 4.0 International License, which permits use, sharing, adaptation, distribution and reproduction in any medium or format, as long as you give appropriate credit to the original author(s) and the source, provide a link to the Creative Commons licence, and indicate if changes were made. The images or other third party material in this article are included in the article's Creative Commons licence, unless indicated otherwise in a credit line to the material. If material is not included in the article's Creative Commons licence and your intended use is not permitted by statutory regulation or exceeds the permitted use, you will need to obtain permission directly from the copyright holder. To view a copy of this licence, visit http://creativecommons.org/licenses/by/4.0/.

\section{References}

Papers of particular interest, published recently, have been highlighted as:

- Of importance

- Of major importance

1. Worm M, Eckermann O, Dolle S, Aberer W, Beyer K, Hawranek $\mathrm{T}$, et al. Triggers and treatment of anaphylaxis: an analysis of 4,000 cases from Germany. Austria Switzerland Dtsch Arztebl Int. 2014;111(21):367-75.

2. Blank S, Pehlivanli S, Methe H, Schmidt-Weber CB, Biedermann T, Horny HP, et al. Fatal anaphylaxis following a hornet sting in a yellow jacket venom-sensitized patient with undetected monoclonal mast cell activation syndrome and without previous history of a systemic sting reaction. J Allergy Clin Immunol Pract. 2020;8(1):401-3 e2.

3. Bilo MB. Anaphylaxis caused by Hymenoptera stings: from epidemiology to treatment. Allergy. 2011;66(Suppl 95):35-7.

4. Sturm GJ, Varga EM, Roberts G, Mosbech H, Bilo MB, Akdis CA, et al. EAACI guidelines on allergen immunotherapy: Hymenoptera venom allergy. Allergy. 2018;73(4):744-64.

5. Hoffman DR. Allergens in Hymenoptera venom. XVI: studies of the structures and cross-reactivities of vespid venom phospholipases. J Allergy Clin Immunol. 1986;78(2):337-43.

6. Hoffman DR. Allergens in Hymenoptera venom XV: the immunologic basis of vespid venom cross-reactivity. J Allergy Clin Immunol. 1985;75(5):611-3.

7. Hoffman DR. Allergens in Hymenoptera venom XIV: IgE binding activities of venom proteins from three species of vespids. J Allergy Clin Immunol. 1985;75(5):606-10.

8. Hoffman DR. Allergens in Hymenoptera venom. XXV: the amino acid sequences of antigen 5 molecules and the structural basis of antigenic cross-reactivity. J Allergy Clin Immunol. 1993;92(5): 707-16.

9. Hoffman DR. Reactions to less common species of fire ants. J Allergy Clin Immunol. 1997;100(5):679-83.

10. Hoffman DR, Wood CL. Allergens in Hymenoptera venom XI. Isolation of protein allergens from Vespula maculifrons (yellow jacket) venom. J Allergy Clin Immunol. 1984;74(1):93-103.

11. King TP, Sobotka AK, Alagon A, Kochoumian L, Lichtenstein LM. Protein allergens of white-faced hornet, yellow hornet, and yellow jacket venoms. Biochemistry. 1978;17(24):5165-74.

12. King TP, Kochoumian L, Lam T. Immunochemical observations of antigen 5, a major venom allergen of hornets, yellowjackets and wasps. Mol Immunol. 1987;24(8):857-64.

13. Gibbs GM, Roelants K, O'Bryan MK. The CAP superfamily: cysteine-rich secretory proteins, antigen 5 , and pathogenesisrelated 1 proteins-roles in reproduction, cancer, and immune defense. Endocr Rev. 2008;29(7):865-97.

14. Bilo MB, Ollert M, Blank S. The role of component-resolved diagnosis in Hymenoptera venom allergy. Curr Opin Allergy Clin Immunol. 2019;19(6):614-22 A current comprehensive review on molecular diagnostics of Hymenoptera venom allergy.

15. Blank S, Bilo MB, Ollert M. Component-resolved diagnostics to direct in venom immunotherapy: important steps towards precision medicine. Clin Exp Allergy. 2018;48(4):354-64 A current comprehensive review on molecular diagnostics of Hymenoptera venom allergy.

16. Jakob T, Müller U, Helbling A, Spillner E. Component resolved diagnostics for hymenoptera venom allergy. Curr Opin Allergy 
Clin Immunol. 2017;17(5):363-72 A comprehensive review on diagnostic approaches in Hymenoptera venom allergy.

17. Ollert M, Blank S. Anaphylaxis to insect venom allergens: role of molecular diagnostics. Curr Allergy Asthma Rep. 2015;15(5):527.

18. Spillner E, Blank S, Jakob T. Hymenoptera allergens: from venom to "venome". Front Immunol. 2014;5:77.

19. Jakob T, Rafei-Shamsabadi D, Spillner E, Müller S. Diagnostics in Hymenoptera venom allergy: current concepts and developments with special focus on molecular allergy diagnostics. Allergo J Int. 2017;26(3):93-105.

20.• Vos B, Köhler J, Müller S, Stretz E, Rueff F, Jakob T. Spiking venom with rVes v 5 improves sensitivity of IgE detection in patients with allergy to Vespula venom. J Allergy Clin Immunol. 2013;131(4):1225-7, 7 e1 An interesting study that demonstrated that spiking of YJV extract with Ves $v 5$ increases diagnostic sensitivity.

21. Ribeiro JM, Francischetti IM. Role of arthropod saliva in blood feeding: sialome and post-sialome perspectives. Annu Rev Entomol. 2003;48:73-88.

22. Ma D, Li Y, Dong J, An S, Wang Y, Liu C, et al. Purification and characterization of two new allergens from the salivary glands of the horsefly. Tabanus Yao Allergy. 2011;66(1):101-9.

23. Quercia O, Emiliani F, Foschi FG, Stefanini GF. The wasp-horsefly syndrome. Eur Ann Allergy Clin Immunol. 2008;40(2):61-3.

24. Sabbah A, Hassoun S, Drouet M, Lauret MG, Doucet M. The waspmosquito syndrome: extension of cross-allergenicity to the horsefly. Allerg Immunol (Paris). 2000;32(1):16-9.

25. Van Vaerenbergh M, Cardoen D, Formesyn EM, Brunain M, Van Driessche G, Blank S, et al. Extending the honey bee venome with the antimicrobial peptide apidaecin and a protein resembling wasp antigen 5. Insect Mol Biol. 2013;22(2):199-210.

26. Radauer C, Nandy A, Ferreira F, Goodman RE, Larsen JN, Lidholm J, et al. Update of the WHO/IUIS allergen nomenclature database based on analysis of allergen sequences. Allergy. 2014;69(4):413-9.

27. King TP, Alagon AC, Kuan J, Sobotka AK, Lichtenstein LM. Immunochemical studies of yellowjacket venom proteins. Mol Immunol. 1983;20(3):297-308.

28. Kosnik M, Korosec P, Silar M, Music E, Erzen R. Wasp venom is appropriate for immunotherapy of patients with allergic reaction to the European hornet sting. Croat Med J. 2002;43(1):25-7.

29. Macchia D, Cortellini G, Mauro M, Meucci E, Quercia O, Manfredi $\mathrm{M}$, et al. Vespa crabro immunotherapy versus Vespula-venom immunotherapy in Vespa crabro allergy: a comparison study in field re-stings. World Allergy Organ J. 2018;11(1):3.

30. Monsalve RI, Vega A, Marques L, Miranda A, Fernandez J, Soriano V, et al. Component-resolved diagnosis of vespid venom-allergic individuals: phospholipases and antigen $5 \mathrm{~s}$ are necessary to identify Vespula or Polistes sensitization. Allergy. 2012;67(4):528-36 A study that showed that using phospholipases A1 and antigen 5 allergens of YJV and PDV facilitates the discrimination of allergies to the two species.

31.• Quercia O, Cova V, Martini M, Cortellini G, Murzilli F, Bignardi $\mathrm{D}$, et al. CAP-inhibition, molecular diagnostics, and Total IgE in the evaluation of Polistes and Vespula double sensitization. Int Arch Allergy Immunol. 2018;177(4):365-9 A recent study that comprehensively demonstrates diagnostic possibilities and limitations in the discrimination of YJV and PDV allergy.

32.• Schiener M, Eberlein B, Moreno-Aguilar C, Pietsch G, Serrano P, McIntyre M, et al. Application of recombinant antigen 5 allergens from seven allergy-relevant Hymenoptera species in diagnostics. Allergy. 2017;72(1):98-108 This study demonstrates the high degree of cross-reactivity between antigen 5 allergens in sIgE measurements as well as in BAT using recombinantly produced antigen 5 allergens of 7 allergy-relevant Hymenoptera species.

33. Sanchez F, Blanca M, Fernandez J, Miranda A, Terrados A, Torres MJ, et al. Comparative study between European and American species of Polistes using sera from European sensitized subjects. Clin Exp Allergy. 1995;25(3):281-7.

34. Severino MG, Campi P, Macchia D, Manfredi M, Turillazzi S, Spadolini I, et al. European Polistes venom allergy. Allergy. 2006;61(7):860-3.

35. Hoffman DR, Sakell RH, Schmidt M. Sol i 1, the phospholipase allergen of imported fire ant venom. J Allergy Clin Immunol. 2005;115(3):611-6.

36. Jeong KY, Yi MH, Son M, Lyu D, Lee JH, Yong TS, et al. IgE reactivity of recombinant Pac c 3 from the Asian needle ant (Pachycondyla chinensis). Int Arch Allergy Immunol. 2016;169(2):93-100.

37. Henriksen A, King TP, Mirza O, Monsalve RI, Meno K, Ipsen H, et al. Major venom allergen of yellow jackets, Ves v 5: structural characterization of a pathogenesis-related protein superfamily. Proteins. 2001;45(4):438-48.

38. Padavattan S, Schmidt M, Hoffman DR, Markovic-Housley Z. Crystal structure of the major allergen from fire ant venom, sol $\mathrm{i}$ 3. J Mol Biol. 2008;383(1):178-85.

39. Müller UR, Johansen N, Petersen AB, Fromberg-Nielsen J, Haeberli G. Hymenoptera venom allergy: analysis of double positivity to honey bee and Vespula venom by estimation of $\operatorname{IgE}$ antibodies to species-specific major allergens Api $\mathrm{m} 1$ and Ves v5. Allergy. 2009;64(4):543-8.

40. Hofmann SC, Pfender N, Weckesser S, Huss-Marp J, Jakob T. Added value of IgE detection to rApi $\mathrm{m} 1$ and rVes $\mathrm{v} 5$ in patients with Hymenoptera venom allergy. J Allergy Clin Immunol. 2011;127(1):265-7.

41. Köhler J, Blank S, Muller S, Bantleon F, Frick M, Huss-Marp J, et al. Component resolution reveals additional major allergens in patients with honeybee venom allergy. J Allergy Clin Immunol. 2014;133(5):1383-9 9 e1-6.

42. Ebo DG, Faber M, Sabato V, Leysen J, Bridts CH, De Clerck LS. Component-resolved diagnosis of wasp (yellow jacket) venom allergy. Clin Exp Allergy. 2013;43(2):255-61.

43. Korosec P, Valenta R, Mittermann I, Celesnik N, Silar M, Zidarn $\mathrm{M}$, et al. High sensitivity of CAP-FEIA rVes v 5 and rVes $\mathrm{v} 1$ for diagnosis of Vespula venom allergy. J Allergy Clin Immunol. 2012;129(5):1406-8.

44. Selb J, Kogovsek R, Silar M, Kosnik M, Korosec P. Improved recombinant Api $\mathrm{m}$ 1- and Ves $\mathrm{v}$ 5-based IgE testing to dissect bee and yellow jacket allergy and their correlation with the severity of the sting reaction. Clin Exp Allergy. 2016;46(4):621-30.

45. Schrautzer C, Bokanovic D, Hemmer W, Lang R, Hawranek T, Schwarz I, et al. Sensitivity and specificity of Hymenoptera allergen components depend on the diagnostic assay employed. J Allergy Clin Immunol. 2016;137(5):1603-5.

46.• Jakob T, Spillner E. Comparing sensitivity of Hymenoptera allergen components on different diagnostic assay systems: comparing apples and oranges? J Allergy Clin Immunol. 2017;139(3):1066-7 A recent study that shows that differences in diagnostic sensitivity of different SIgE assay platforms are due to the calibration approaches that are used.

47. Szecsi PB, Stender S. Comparison of immunoglobulin E measurements on IMMULITE and ImmunoCAP in samples consisting of allergen-specific mouse-human chimeric monoclonal antibodies towards allergen extracts and four recombinant allergens. Int Arch Allergy Immunol. 2013;162(2):131-4. 
48. Wood RA, Segall N, Ahlstedt S, Williams PB. Accuracy of IgE antibody laboratory results. Ann Allergy Asthma Immunol. 2007;99(1):34-41.

49. Michel J, Brockow K, Darsow U, Ring J, Schmidt-Weber CB, Grunwald T, et al. Added sensitivity of component-resolved diagnosis in hymenoptera venom-allergic patients with elevated serum tryptase and/or mastocytosis. Allergy. 2016;71(5):651-60.

50. Sturm GJ, Bilo MB, Bonadonna P, Hemmer W, Caruso B, Bokanovic D, et al. Ves v 5 can establish the diagnosis in patients without detectable specific IgE to wasp venom and a possible north-south difference in Api m 1 sensitization in Europe. J Allergy Clin Immunol. 2012;130(3):817-author reply 8-9, 817; author reply 819 .

51. Müller U, Schmid-Grendelmeier P, Hausmann O, Helbling A. IgE to recombinant allergens Api $\mathrm{m} 1$, Ves v 1, and Ves v 5 distinguish double sensitization from crossreaction in venom allergy. Allergy. 2012;67(8):1069-73.

52. Gattinger P, Lupinek C, Kalogiros L, Silar M, Zidarn M, Korosec $\mathrm{P}$, et al. The culprit insect but not severity of allergic reactions to bee and wasp venom can be determined by molecular diagnosis. PLoS One. 2018;13(6):e0199250 A recent study that demonstrates the potential of molecular diagnostics to differentiate between $\mathrm{HBV}$ and YJV allergy. Moreover it is shown that the severity of the sting reaction is not associated with parameters obtained by CRD.

53. Vos B, van Anrooij B, van Doormaal JJ, Dubois AEJ, Oude Elberink JNG. Fatal anaphylaxis to yellow jacket stings in Mastocytosis: options for identification and treatment of at-risk patients. J Allergy Clin Immunol Pract. 2017;5(5):1264-71.

54. Hamilton RG, Oppenheimer J. Serological IgE analyses in the diagnostic algorithm for allergic disease. J Allergy Clin Immunol Pract. 2015;3(6):833-40 quiz 41-2.

55. Ollert M, Weissenbacher S, Rakoski J, Ring J. Allergen-specific IgE measured by a continuous random-access immunoanalyzer: interassay comparison and agreement with skin testing. Clin Chem. 2005;51(7):1241-9.

56. Perez-Riverol A, Dos Santos-Pinto JRA, Lasa AM, Palma MS, Brochetto-Braga MR. Wasp venomic: unravelling the toxins arsenal of Polybia Paulista venom and its potential pharmaceutical applications. J Proteome. 2017;161:88-103.

57. Bazon ML, Perez-Riverol A, Dos Santos-Pinto JRA, Fernandes LGR, Lasa AM, Justo-Jacomini DL, et al. Heterologous expression, purification and immunoreactivity of the antigen 5 from Polybia paulista wasp venom. Toxins (Basel). 2017;9(9) In this study, antigen 5 of Polybia paulista, an important elicitor of venom allergy in South America, was recombinantly produced. This antigen 5 allergen is an interesting candidate for adequate diagnostics of Polybia venom allergy.

58. Perez-Riverol A, Campos Pereira FD, Musacchio Lasa A, Romani Fernandes LG, Santos-Pinto JR, Justo-Jacomini DL, et al. Molecular cloning, expression and IgE-immunoreactivity of phospholipase A1, a major allergen from Polybia paulista (Hymenoptera: Vespidae) venom. Toxicon. 2016;124:44-52.

59. Lambert C, Birnbaum J, Dzviga C, Hutt N, Apoil PA, Bienvenu F, et al. Antigen 5-spiked Vespula and Polistes venom extracts for Vespid allergy diagnostics: a French multicenter study. Ann Allergy Asthma Immunol. 2018;120(4):435-7.

60. Yoshida N, Hirata H, Watanabe M, Sugiyama K, Arima M, Fukushima Y, et al. Improved sensitivity to venom specificimmunoglobulin $\mathrm{E}$ by spiking with the allergen component in Japanese patients suspected of Hymenoptera venom allergy. Allergol Int. 2015;64(3):248-52.

61. Bokanovic D, Schwarz I, Wutte N, Komericki P, Aberer W, Sturm GJ. Specificity of conventional and Ves v 5-spiked venom decreases with increasing total IgE. J Allergy Clin Immunol. 2014;134(3):739-41.
62. Jappe U, Raulf-Heimsoth M, Hoffmann M, Burow G, HubschMuller C, Enk A. In vitro hymenoptera venom allergy diagnosis: improved by screening for cross-reactive carbohydrate determinants and reciprocal inhibition. Allergy. 2006;61(10):1220-9.

63. Hemmer W, Focke M, Kolarich D, Dalik I, Gotz M, Jarisch R. Identification by immunoblot of venom glycoproteins displaying immunoglobulin E-binding $\mathrm{N}$-glycans as cross-reactive allergens in honeybee and yellow jacket venom. Clin Exp Allergy. 2004;34(3):460-9.

64. Hemmer W, Focke M, Kolarich D, Wilson IB, Altmann F, Wohrl S, et al. Antibody binding to venom carbohydrates is a frequent cause for double positivity to honeybee and yellow jacket venom in patients with stinging-insect allergy. J Allergy Clin Immunol. 2001;108(6):1045-52.

65. Sturm GJ, Hemmer W, Hawranek T, Lang R, Ollert M, Spillner E, et al. Detection of IgE to recombinant Api $\mathrm{m} 1$ and $\mathrm{rVes} v 5$ is valuable but not sufficient to distinguish bee from wasp venom allergy. J Allergy Clin Immunol. 2011;128(1):247-8 author reply 8.

66.• Selb J, Bidovec Stojkovic U, Bajrovic N, Kopac P, Erzen R, Zidarn $\mathrm{M}$, et al. Limited ability of recombinant Hymenoptera venom allergens to resolve IgE double sensitization. J Allergy Clin Immunol Pract. 2018;6(6):2118-20 A recent study on the limited ability of CRD to identify the clinically relevant venom in patients double-sensitized to venom extracts due to the lack of crossreactive allergen pairs and the presence of double sensitization also with recombinant venom components.

67. Blanca M, Garcia F, Miranda A, Carmona MJ, Garcia J, Fernandez $\mathrm{J}$, et al. Determination of $\operatorname{IgE}$ antibodies to Polistes dominulus, Vespula germanica and Vespa crabro in sera of patients allergic to vespids. Allergy. 1991;46(2):109-14.

68. Grant JA, Rahr R, Thueson DO, Lett-Brown MA, Hokanson JA, Yunginger JW. Diagnosis of Polistes wasp hypersensitivity. J Allergy Clin Immunol. 1983;72(4):399-406.

69. Galindo-Bonilla PA, Galan-Nieto A, Alfaya-Arias T, GarciaRodriguez C, de la Roca-Pinzon F, Feo-Brito F. Componentresolved diagnosis in vespid venom-allergic individuals. Allergol Immunopathol (Madr). 2015;43(4):398-402.

70. Caruso B, Bonadonna P, Severino MG, Manfredi M, Dama A, Schiappoli M, et al. Evaluation of the IgE cross-reactions among vespid venoms. A possible approach for the choice of immunotherapy. Allergy. 2007;62(5):561-4.

71. Savi E, Peveri S, Makri E, Pravettoni V, Incorvaia C. Comparing the ability of molecular diagnosis and CAP-inhibition in identifying the really causative venom in patients with positive tests to Vespula and Polistes species. Clin Mol Allergy. 2016;14:3.

72. Caruso B, Bonadonna P, Bovo C, Melloni N, Lombardo C, Senna $\mathrm{G}$, et al. Wasp venom allergy screening with recombinant allergen testing. Diagnostic performance of rPol d 5 and rVes v 5 for differentiating sensitization to Vespula and Polistes subspecies. Clin Chim Acta. 2016;453:170-3.

73. Buck M, Marshall SA, Cheung DKB. Identification atlas of the Vespidae (Hymenoptera, Aculeata) of the northeastern Nearctic Region. Can J Arthropod Identif. 2008;5:1-492.

74. Cervo R, Zacchi F, Turillazzi S. Polistes dominulus (Hymenoptera, Vespidae) invading North America: some hypotheses for its rapid spread. Insect Soc. 2000;47(2):155-7.

75. Eardley C, Koch F, Wood AR. Polistes dominulus (Christ, 1791) (Hymenoptera: Polistinae: Vespidae) newly recorded from South Africa : short communication. Afr Entomol. 2009;17(2):226-7.

76. Sackmann P, Villacide JM, Corley J. Presencia de una nueva avispa social exótica, Polistes dominulus (Hymenoptera: Vespidae) en la Patagonia argentina. Revista de la Sociedad Entomológica Argentina. 2003;62:72-4.

77. Smit J. De Veldwespen Polistes dominulus en P. biglumis op in Nederland (Hymenoptera: Vespidae). Nederlandse Faunistische Mededelingen. 2003;18:81-8. 
78. Perez-Riverol A, Palma MS, Jakob T. Current challenges in diagnostics of insect venom allergy. Allergo J Int. 2020. https://doi.org/ 10.18176/jiaci.0498 An interesting review on current limitations and future needs in CRD of Hymenoptera venom allergy.

79. Balzer L, Pennino D, Blank S, Seismann H, Darsow U, Schnedler $\mathrm{M}$, et al. Basophil activation test using recombinant allergens: highly specific diagnostic method complementing routine tests in wasp venom allergy. PLoS One. 2014;9(10):e108619.

80. Bohle B, Zwolfer B, Fischer GF, Seppala U, Kinaciyan T, Bolwig $\mathrm{C}$, et al. Characterization of the human $\mathrm{T}$ cell response to antigen 5 from Vespula vulgaris (Ves v 5). Clin Exp Allergy. 2005;35(3): 367-73.

81. dos Santos-Pinto JR, dos Santos LD, Arcuri HA, da Silva Menegasso AR, Pego PN, Santos KS, et al. B-cell linear epitopes mapping of antigen-5 allergen from Polybia paulista wasp venom. J Allergy Clin Immunol. 2015;135(1):264-7.

82. Suck R, Weber B, Kahlert H, Hagen S, Cromwell O, Fiebig H. Purification and immunobiochemical characterization of folding variants of the recombinant major wasp allergen Ves $\mathrm{v} 5$ (antigen 5). Int Arch Allergy Immunol. 2000;121(4):284-91.

83. Hoffman DR, McDonald CA. Allergens in hymenoptera venom. IX. Species specificity to Polistes (paper wasp) venoms. Ann Allergy. 1982;48(2):82-6.

84. Pantera B, Hoffman DR, Carresi L, Cappugi G, Turillazzi S, Manao $\mathrm{G}$, et al. Characterization of the major allergens purified from the venom of the paper wasp Polistes gallicus. Biochim Biophys Acta. 2003;1623(2-3):72-81.

85. Potiwat R, Tanyaratsrisakul S, Maneewatchararangsri S, Manuyakorn W, Rerkpattanapipat T, Samung Y, et al. Solenopsis geminata (tropical fire ant) anaphylaxis among Thai patients: its allergens and specific IgE-reactivity. Asian Pac J Allergy Immunol. 2018;36(2):101-8.

86. Hoffman DR, Smith AM, Schmidt M, Moffitt JE, Guralnick M. Allergens in Hymenoptera venom. XXII. Comparison of venoms from two species of imported fire ants, Solenopsis invicta and richteri. J Allergy Clin Immunol. 1990;85(6):988-96.

87. Hoffman DR, Jacobson RS, Zerboni R. Allergens in hymenoptera venom. XIX. Allergy to Vespa crabro, the European hornet. Int Arch Allergy Appl Immunol. 1987;84(1):25-31.

88. Hiller R, Laffer S, Harwanegg C, Huber M, Schmidt WM, Twardosz A, et al. Microarrayed allergen molecules: diagnostic gatekeepers for allergy treatment. FASEB J. 2002;16(3):414-6.

89. Hoffman DR, McDonald CA. Allergens in hymenoptera venom. VIII. Immunologic comparison of venoms from six species of Vespula (yellow jackets). Ann Allergy. 1982;48(2):78-81.

Publisher's Note Springer Nature remains neutral with regard to jurisdictional claims in published maps and institutional affiliations. 